\title{
Electric field induced critical points and field gradient by trapped electrons in Li-doped $\mathrm{KTa}_{1-\mathrm{x}} \mathrm{Nb}_{\mathrm{x}} \mathrm{O}_{3}$ single crystals
}

M. M. Rahaman, T. Imai, T. Sakamoto, and S. Kojima

Citation: Appl. Phys. Lett. 111, 032904 (2017); doi: 10.1063/1.4993915

View online: http://dx.doi.org/10.1063/1.4993915

View Table of Contents: http://aip.scitation.org/toc/apl/111/3

Published by the American Institute of Physics

\section{Articles you may be interested in}

Material descriptors for morphotropic phase boundary curvature in lead-free piezoelectrics

Applied Physics Letters 111, 032907 (2017); 10.1063/1.4990955

Pressure driven depolarization behavior of $\mathrm{Bi}_{0.5} \mathrm{Na}_{0.5} \mathrm{TiO}_{3}$ based lead-free ceramics

Applied Physics Letters 110, 212901 (2017); 10.1063/1.4984088

Enhanced resistive memory in $\mathrm{Nb}$-doped $\mathrm{BaTiO}_{3}$ ferroelectric diodes

Applied Physics Letters 111, 032902 (2017); 10.1063/1.4993938

Polarization dependent ferroelectric photovoltaic effects in BFTO/CuO thin films

Applied Physics Letters 111, 032901 (2017); 10.1063/1.4985563

Field-driven electro-optic dynamics of polar nanoregions in nanodisordered $\mathrm{KTa}_{1-\mathrm{x}} \mathrm{Nb}_{\mathrm{x}} \mathrm{O}_{3}$ crystal

Applied Physics Letters 111, 012903 (2017); 10.1063/1.4991357

Large flexoelectricity in $\mathrm{Al}_{2} \mathrm{O}_{3}$-doped $\mathrm{Ba}\left(\mathrm{Ti}_{0.85} \mathrm{Sn}_{0.15}\right) \mathrm{O}_{3}$ ceramics

Applied Physics Letters 110, 192903 (2017); 10.1063/1.4983195

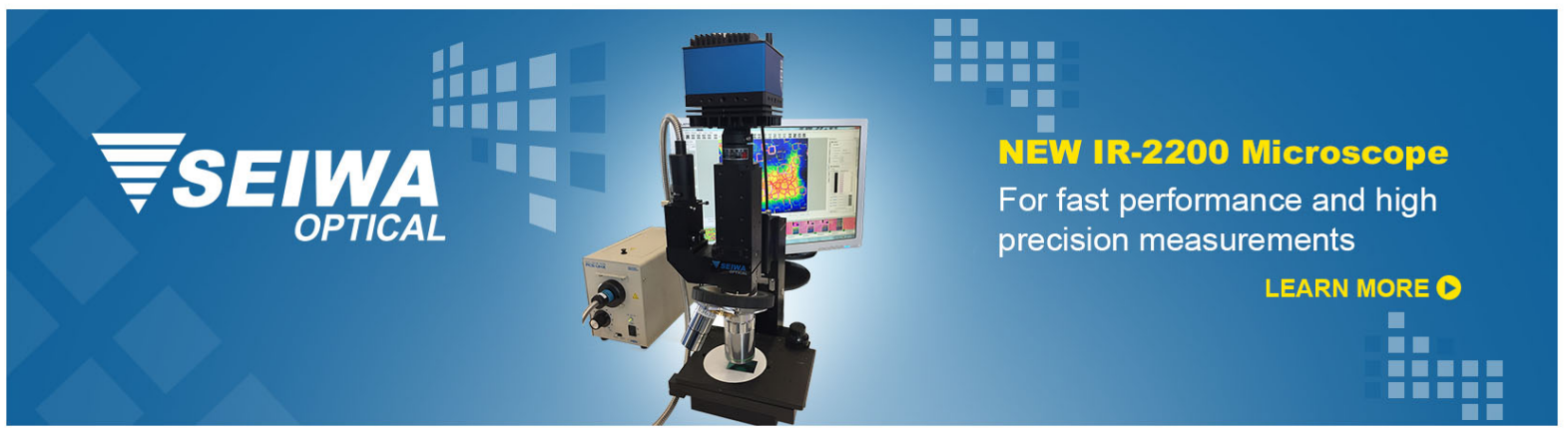




\title{
Electric field induced critical points and field gradient by trapped electrons in $\mathrm{Li}$-doped $\mathrm{KTa}_{1-x} \mathrm{Nb}_{x} \mathrm{O}_{3}$ single crystals
}

\author{
M. M. Rahaman, ${ }^{1,2, a)}$ T. Imai, ${ }^{3}$ T. Sakamoto, ${ }^{3}$ and S. Kojima ${ }^{1, b)}$ \\ ${ }^{1}$ Graduate School of Pure and Applied Sciences, University of Tsukuba, Tsukuba, Ibaraki 305-8573, Japan \\ ${ }^{2}$ Department of Materials Science and Engineering, University of Rajshahi, Rajshahi 6205, Bangladesh \\ ${ }^{3}$ NTT Device Innovation Center, Nippon Telegraph and Telephone Corporation, Atsugi, Kanagawa 243-0198, \\ Japan
}

(Received 30 March 2017; accepted 1 July 2017; published online 18 July 2017)

\begin{abstract}
The effects of electric field and trapped electrons on a ferroelectric phase transition of the 5\%Li-doped $\mathrm{KTa}_{0.74} \mathrm{Nb}_{0.26} \mathrm{O}_{3}$ crystals were investigated by micro-Brillouin scattering and dielectric measurements. In micro-Brillouin scattering, the remarkable changes of the central peak (CP) intensity were observed at the paraelectric cubic to ferroelectric tetragonal phase transition. The critical electric fields to induce the paraelectric to ferroelectric phase transition were found to shift to higher values with increasing temperature. From these $\mathrm{CP}$ results, we estimated lines of critical points for phase transitions including critical end point $(\mathrm{CEP}),(E, T)=\left(1.6 \mathrm{kV} / \mathrm{cm}, T_{\mathrm{C}-\mathrm{T}}+3.4^{\circ} \mathrm{C}\right)$ in the composition-temperature-electric field $(x-T-E)$ phase diagram. The clear difference of the CEP was observed between Brillouin result at a fixed small area and dielectric result averaged over all area of a sample. It can be caused by the field gradient, which is induced by the trapped electrons. The existence of gradient of electric field was also discussed using the observed result of position dependence of the CP intensity. Published by AIP Publishing. [http://dx.doi.org/10.1063/1.4993915]
\end{abstract}

The electro-optic (EO) devices such as an optical beam deflector have been used for light detection and ranging system (LIDAR) and laser display. The Li-doped $\mathrm{KTa}_{1-x} \mathrm{Nb}_{x} \mathrm{O}_{3}$ (KTN) single crystals exhibit huge electro-optic (EO) effect, ${ }^{1,2}$ and excellent photorefractive effect, ${ }^{3}$ which make them particularly attractive for the application to optical devices such as optical beam deflector. ${ }^{4}$ Recently, a new optical beam deflection phenomenon by the space charge was observed in KTN crystals. ${ }^{4,5}$ The space charge is formed by applying a voltage to a pair electrodes, and electrons are injecting into the KTN crystals from the cathode. ${ }^{4,5}$ Hence, at first the space charge forms an electric field distribution, and then the distribution of refractive index occurs by EO effect. This distribution of refractive index bends a beam of light, and the deflector can achieve deflection angles exceeding several degrees that are much larger than those obtained from conventional EO effect. ${ }^{6}$ However, the electrons, which were injecting into a KTN crystal, are trapped by the localized states in the crystal, and they are stabilized. Recently, Nakamura et al. observed the effect of the space charge on the optical beam deflection and reported that the space charges induced electric fields are not uniform inside a KTN crystal. ${ }^{4}$ Moreover, the significant increase in the permittivity and capacitance of a Li-doped KTN crystal was observed by injecting electrons. ${ }^{7}$

Another important feature in Li-doped KTN is the offcenter displacements of $\mathrm{Li}$ and $\mathrm{Nb}$ ions at the $\mathrm{A}$ and $\mathrm{B}$-sites, respectively, and therefore, they induce polar nanoregions (PNRs). ${ }^{8,9}$ The off-center displacements of $\mathrm{Nb}$ ions at B-site in KTN was explained by eight site model in which $\mathrm{Nb}$ ions displace among the equivalent [111] directions, ${ }^{10}$ while the

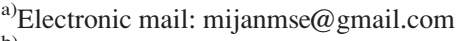

b)Electronic mail: kojima@ims.tsukuba.ac.jp
}

substitutional Li ions occupy one of six off-center sites along the [100] directions at the A-site. ${ }^{11,12}$ The existence of PNRs in KTN and Li-doped KTN was reported by various measurements. $^{13-19}$ In the cubic phase, the PNRs are characterized by two temperatures. First, the Burns temperature, $T_{\mathrm{B}}$, at which the dynamic PNRs begin to appear. ${ }^{20}$ Second, the intermediate temperature, $T^{*}$, at which dynamic-to-static transition of PNRs starts. ${ }^{13-16}$

Due to the giant electro-mechanical response in the vicinity of the critical end point $(\mathrm{CEP})^{21}$ the study of the temperaturefield phase diagram is the current topics in advanced materials such as ferroelectric materials. Aftabuzzaman and Kojima also reported that the CEP is the driving mechanism for the polarization rotation and the related giant electromechanical response of relaxor ferroelectrics. ${ }^{22}$ Recently, the temperature-field phase diagram concluding the $\mathrm{CEP}(E, T)=\left(3.3 \mathrm{kV} / \mathrm{cm}, T_{\mathrm{C}-\mathrm{T}}+6^{\circ} \mathrm{C}\right)$ was investigated of a 5\%Li-doped KTN with $x=0.27$ (KLTN/ $5 / 27$ ) single crystal with no injected electrons content. ${ }^{7}$ However, there is no report regarding the effect of trapped electrons on the CEP of the Li-doped KTN crystals, which is crucial for the application of this material as electromechanical devices.

In this study, the electric field effects on a ferroelectric phase transitions of a $5 \% \mathrm{Li}$-doped KTN was investigated by the Brillouin scattering and dielectric measurements. The effects of trapped electrons were discussed by the difference of the CEP results between Brillouin and dielectric measurements. The gradient of the electric field inside the crystal was discussed on the basis of the results of the position dependence of the central peak $(\mathrm{CP})$ intensity.

The 5\% Li-doped $\mathrm{KTa}_{1-x} \mathrm{Nb}_{x} \mathrm{O}_{3}$ with $x=0.26(\mathrm{KLTN} / 5 / 26)$ single crystals used in this present investigation were gown at NTT Corporation by the top seeded solution growth (TSSG) technique. The typical sample dimensions were 3.18 $\times 1.33 \times 1.19 \mathrm{~mm}^{3}$. A Sandercock-type $3+3$ passes tandem 
Fabry-Perot interferometer combined with a modified optical microscope (Olympus BX60) was used to measure the Brillouin spectra in the frequency range of -65 to $65 \mathrm{GHz}$ for the laser beam of $532 \mathrm{~nm}$ at a backscattering geometry. ${ }^{23} \mathrm{~A}$ pair of titanium electrodes was used to inject electrons into the crystals by applying voltage. The electrodes were deposited on the (100) faces to apply the electric field along [100] direction. The temperature dependence of the dielectric properties under the constant electric field was measured using a LCR meter (Agilent 4263B) with an external voltage bias fixture. The temperature of the sample was controlled by a THMS600 (Linkam) heating/cooling stage with a stability $\pm 1{ }^{\circ} \mathrm{C}$.

The typical Brillouin spectra of the KLTN/5/26 single crystal at selected electric fields under a constant temperature in a narrow frequency range are shown in Fig. 1. As can be seen in Fig. 1, each spectrum consists of a longitudinal acoustic (LA) mode, a transverse acoustic (TA) mode, and a central peak $(\mathrm{CP})$. The paraelectric cubic to ferroelectric tetragonal phase transition temperature, $T_{\mathrm{C}-\mathrm{T}}=23.6^{\circ} \mathrm{C}$ of the $\mathrm{KLTN} / 5$ / 26 single crystal, was determined by the significant softening of the LA frequency shift upon zero field heating (ZFH) [Fig. 2(b)] and remarkable increase in the dielectric constant measured at $1 \mathrm{kHz}$ (Fig. 4). It is apparent that the TA mode is intense even in the cubic phase (Fig. 1), which is forbidden at a back scattering geometry according to the Brillouin selection rule. ${ }^{24}$ Therefore, the existence of the TA mode in the cubic phase may reflect the local breaking of symmetry. Recently, Rahaman et al. reported the breaking of local symmetry and the microscopic origin of the CP in the cubic phase of a high quality KLTN/5/27 crystal by the observation of $E(x, y)$ symmetry of PNRs with the rhombohedral $R 3 m$ symmetry using Raman scattering. ${ }^{15}$ It is important to note that the intensity of the LA mode is relatively weak and it exhibits asymmetric nature (Fig. 1). The mode coupling between LA mode and CP may causes the intensity transfer from the LA mode into $\mathrm{CP}$, and the intensity of LA mode becomes weak.

The Brillouin scattering spectra and electric voltage dependence of the CP intensity of the KLTN/5/26 crystal are shown in Figs. 1 and 2(a), respectively. The remarkable changes of the $\mathrm{CP}$ intensity were observed at the critical voltages, indicating

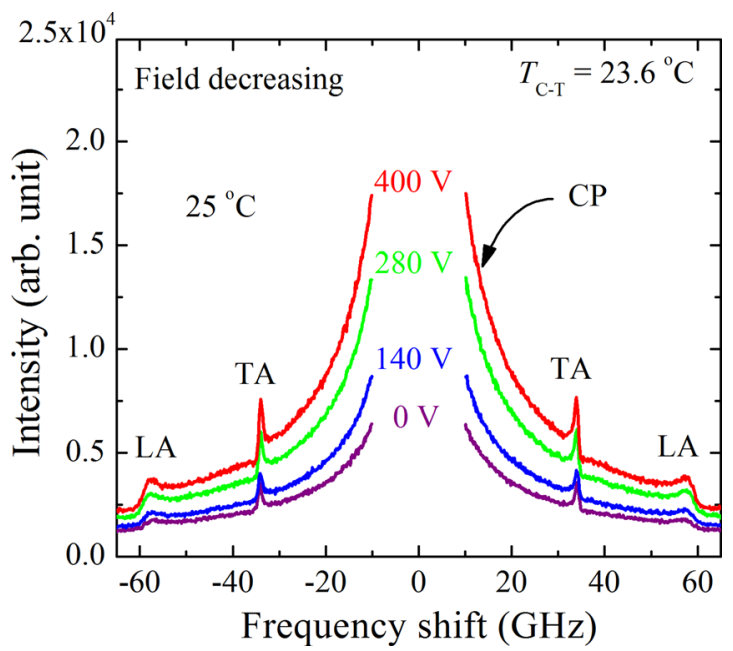

FIG. 1. Brillouin scattering spectra of a KLTN/5/26 single crystal at selected electric voltages measured with FSR $=75 \mathrm{GHz}$ under a constant temperature.
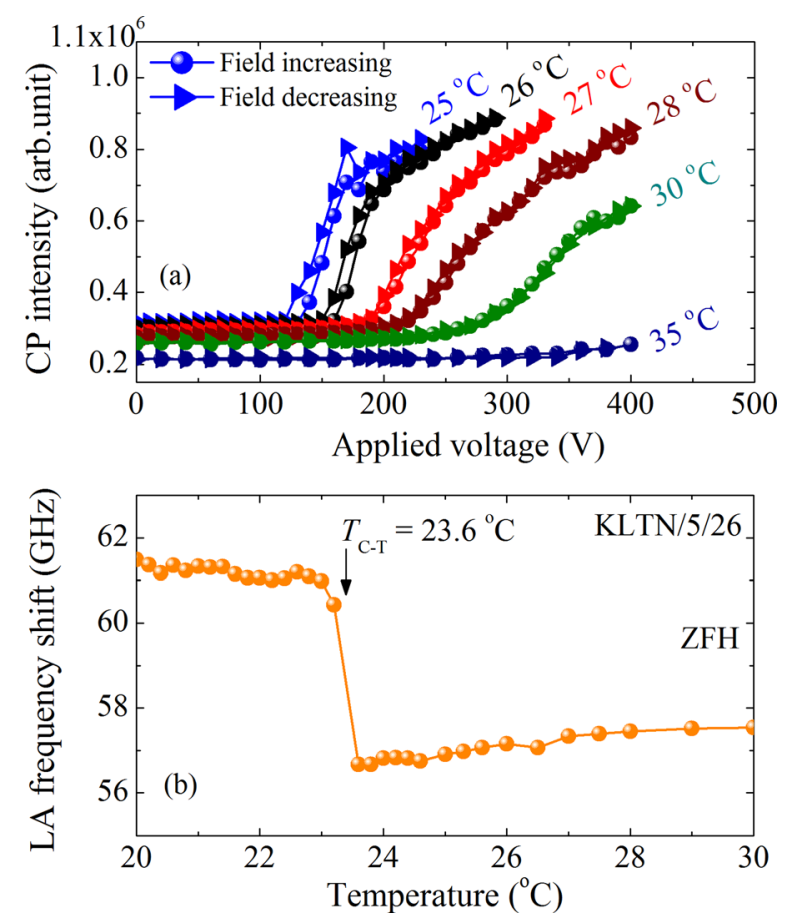

FIG. 2. (a) Electric voltage dependence of the CP intensity of the KLTN/5/ 26 crystal at selected temperatures. (b) The frequency shift of the LA mode as a function of temperature.

the paraelectric cubic to ferroelectric tetragonal phase transition. In relaxor ferroelectrics, it is believed that most of the dynamic PNRs become static in the vicinity of the $T_{\mathrm{C}-\mathrm{T}}$. Therefore, the significant increase of the $\mathrm{CP}$ intensity at the critical voltage may be caused by the alignment of randomly oriented static and dynamic PNRs along the direction of the applied voltage results in a [100] time-averaged polarizations, and crystal transform into a lower tetragonal $P 4 \mathrm{~mm}$ symmetry.

It was found that the paraelectric to ferroelectric phase transition on heating and the ferroelectric to paraelectric phase transition on cooling occurred at the electric voltages $V_{\mathrm{C} 1}$ and $V_{\mathrm{C} 2}$, respectively. The small hysteresis behavior of the CP intensity was observed between heating and cooling runs (Fig. 2), which reflects the slightly first order nature of the ferroelectric phase transition. The critical electric voltages to induce the paraelectric to ferroelectric phase transition were found to shift to higher values with increasing temperature as shown in Fig. 3. However, the shift of $V_{\mathrm{C} 2}$ is larger than the shift of $V_{\mathrm{C} 1}$; therefore, $V_{\mathrm{C} 2}$ and $V_{\mathrm{C} 1}$ merges at around $(V, T)=\left(190 \mathrm{~V}, T_{\mathrm{C}-\mathrm{T}}+3.4^{\circ} \mathrm{C}\right)$, which is equivalent to $(E, T)=\left(1.3 \mathrm{kV} / \mathrm{cm}, T_{\mathrm{C}-\mathrm{T}}+3.4{ }^{\circ} \mathrm{C}\right)$ as shown in Fig. 3 . The hysteresis behavior gradually diminished to zero as the voltage was increased beyond that of this point. This point was identified as the critical end point (CEP) of the KLTN/5/ 26 crystal, and the first order nature of the ferroelectric phase transition changes into the second order one at this point. ${ }^{7}$ The value of the CEP, $(E, T)=\left(3.3 \mathrm{kV} / \mathrm{cm}, T_{\mathrm{C}-\mathrm{T}}+6{ }^{\circ} \mathrm{C}\right)$, of the KLTN/5/27 crystal was reported in which there was no injected electrons into the crystal. ${ }^{7}$ Therefore, the lower value of the averaged electric field, $E_{\mathrm{av}}=1.7 \mathrm{kV} / \mathrm{cm}$, observed in CEP of the KLTN/5/26 crystal can be caused by the space charge induced electric field, which is generated by the injected and/or trapped electrons. 


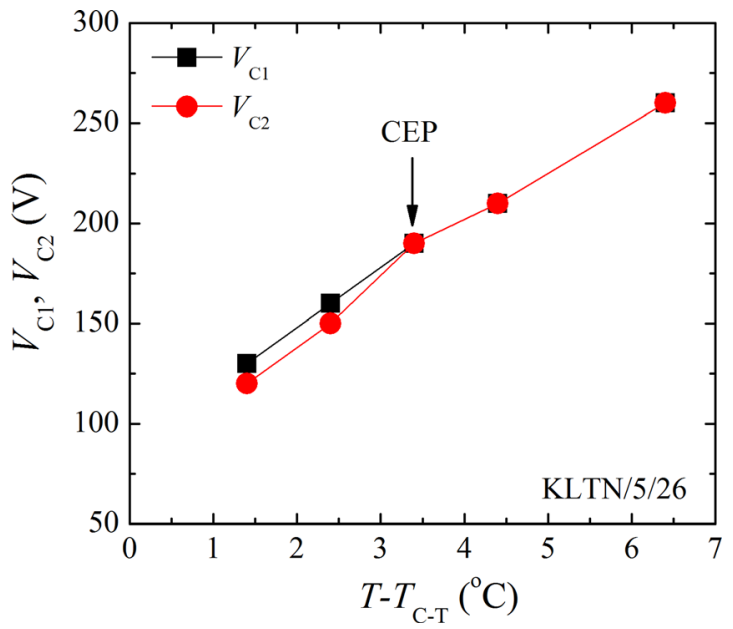

FIG. 3. Critical electric voltages of a phase transition, $V_{\mathrm{C} 1}$ is for the change from paraelectric to ferroelectric phase and $V_{\mathrm{C} 2}$ for the change from ferroelectric to paraelectric phase, as a function of temperature.

In order to look into the effect of injected and/or trapped electrons on the CEP, the dielectric properties of the KLTN/ $5 / 26$ crystal were examined as a function of temperature at constant electric voltages as shown in Fig. 4. The real part of the dielectric constant shows a sharp peak reflecting the transition from ferroelectric to paraelectric phases at about $T_{\mathrm{C}-\mathrm{T}}$ at selected electric voltages (Fig. 4). The drastic change of the dielectric constant near $T_{\mathrm{C}-\mathrm{T}}$ at $0 \mathrm{~V}$ is in good agreement with the elastic anomaly observed at $\mathrm{ZFH}$. The slightly broad peak showing around $T_{\mathrm{C}-\mathrm{T}}$ at $V=0 \mathrm{~V}$ changes into a very sharp phase transition with approaching the CEP with $V_{\mathrm{av}}=154.3 \mathrm{~V}$ or $E_{\mathrm{av}}=1.3 \mathrm{kV} / \mathrm{cm}$. The sharp phase transition of the $(1-x) \mathrm{Pb}\left(\mathrm{Mg}_{1 / 3} \mathrm{Nb}_{2 / 3} \mathrm{O}_{3}-\mathrm{PbTiO}_{3}\right.$ (PMN- $\left.x \mathrm{PT}\right)$ system was clearly observed in the vicinity of the CEP. ${ }^{21,22}$ The conventional dielectric spectroscopy only provides the average value of dielectric constant of the investigated specimen. However, the combination of optical microscope and microBrillouin scattering spectroscopy is possible to measure a very small area of a specimen to clarify the local heterogeneity. ${ }^{23}$ Hence, the difference of the CEP between Brillouin and dielectric measurements may due to field gradient, which is induced by the trapped electrons.
In order to clarify the effect of trapped electrons on the critical voltages, we also study the position dependence of the Brillouin scattering spectra of the KLTN/5/26 crystal. The schematic illustration of three positions in the crystal is shown in Fig. 5(a). The space charge field, $E(x)$, and charge density, $\rho$, are related by Gauss's law as follows: ${ }^{5,7}$

$$
\frac{\partial E(x)}{\partial x}=\rho / \varepsilon
$$

Here, $x$ is the distance from the cathode. We only consider $x$ dependence and ignore the $y$ - and z-dependences. This approximation is valid assuming the sample structure that consists of a KTN plate with high permittivity and electrodes, which covered the entire surface of the largest faces. Equation (1) shows that the electric field has the special distribution because of charge.

If we consider a spatially uniform charge density, the electric field distribution with an applied voltage can be expressed as

$$
E(x)=(\rho / \varepsilon) x+E_{0},
$$

where $\varepsilon$ is the spatial distribution permittivity. ${ }^{7} E_{0}$ is a constant depending on the $E(x)$ profile and the potential difference between the electrodes. $E_{0}$ is zero when the potential difference is zero. Since the injected electrons are bound strongly and the space charge is stable, the scenario of electric field distribution with the $\rho$ changes at $0 \mathrm{~V}$. As the electric field integrated along the $x$ direction equals to the voltage, it holds that

$$
V=-\int_{0}^{\mathrm{d}} E \mathrm{~d} x
$$

With the condition shown in Eq. (3), $E_{0}$ in Eq. (1) is deduced as

$$
E_{0}=-(\rho d / 2 \varepsilon)-(V / d) \text {. }
$$

Thus, the Eq. (2) can be expressed as

$$
E=(\rho / \varepsilon)[x-(d / 2)]-(V / d) .
$$

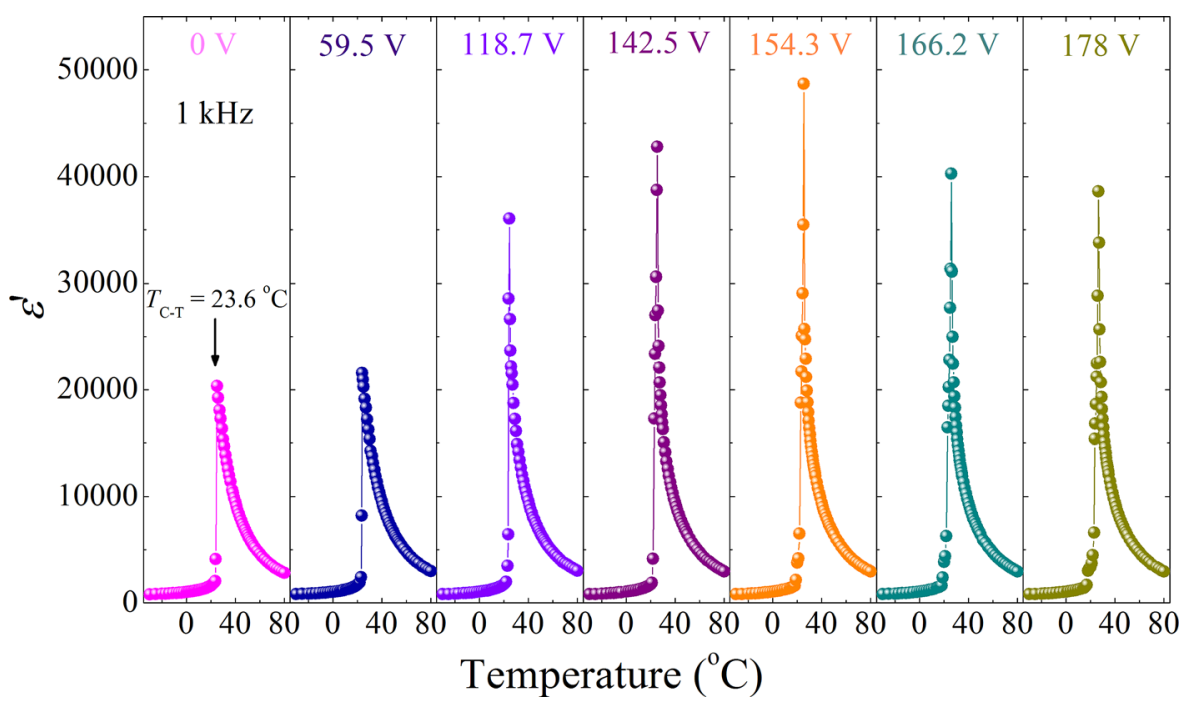

FIG. 4. Temperature dependence of the dielectric constants measured at $1 \mathrm{kHz}$ under the DC biasing voltages. 

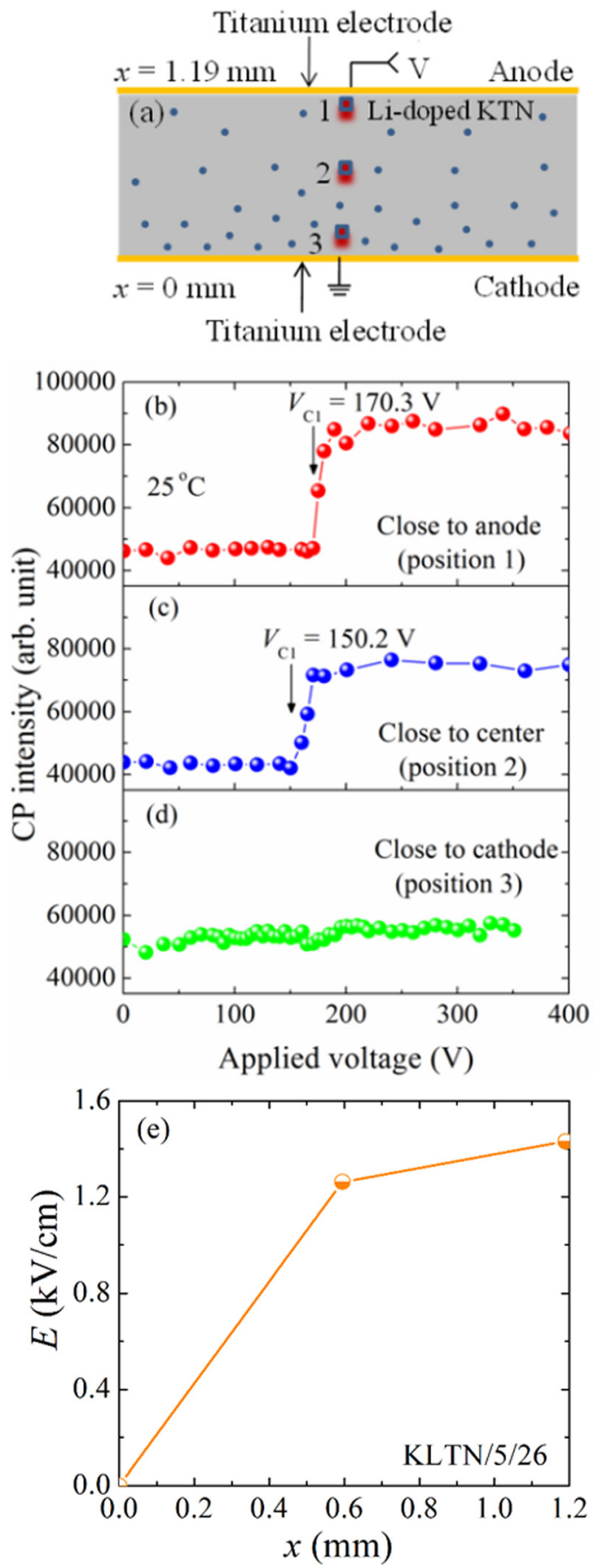

FIG. 5. (a) Schematic illustration of three positions in the KLTN/5/26 crystal. The CP intensity observed at (b) close to anode (position 1), (c) close to center (position 2), and (d) close to cathode (position 3) as a function of electric voltage measured at $25^{\circ} \mathrm{C}$. (e) The position dependence of the critical electric field of the KLTN/5/26 crystal.

Here, the origin of $x$ is taken at the cathode and $d$ is the thickness of a sample. Equation (5) shows that the zero point of the electric field is at the center of a crystal when $V=0$. On the other hand, when the electron injecting voltage is low, $\rho$ is not uniform, and the electron density tends to be large near the cathode compared with the part near the anode. Then, the zero point electric field at $V=0$ is slightly shifted towards the cathode. In the present investigation, we are mainly concerned about the electric field distribution in KLTN/5/26 crystal by injecting electrons with applied voltages. From Eq. (2), it is clear that the absolute value of the electric field is almost zero at the cathode, and it increases with the distance from the cathode and becomes the maximum at the anode. However, an external electric field distorts the lattice because of the electrostrictive effect and the lattice symmetry becomes tetragonal. Thus, under the moderate electric field, the crystal part near the anode tends to become ferroelectric [Fig. 5(b)] because the electric field is strong, whereas the part near the cathode tends to remain paraelectric [Fig. 5(d)]. It is important to note that the value of critical field near anode is somewhat higher than that of the value near center (Fig. 5). These results are similar to those observed in non-doped KTN reported in Ref. 5. In order to have a better understanding of the distribution of charges, we also study the plot of the position dependence of the critical electric field of the KLTN/5/26 crystal. For simplicity, we assume the position $x=0$ (close to cathode), $x=d / 2$ (close to center), and $x=d$ (close to anode) and plot the position dependence of the critical field of the KLTN/5/ 26 crystal as shown in Fig. 5(e). It is difficult to explain the actual nature of the electric field inside the crystal because of small number of observation position. However, the slight deviation from the linear behavior of the electric field can be observed based on the results of the position dependence of the CP intensity [Fig. 5(e)]. Therefore, it is suggested that the deviation from the linear behavior may due to inhomogeneous distribution of charges, which was injected into the crystal with applied voltages. The discrepancy of the value of the capacitance between observed and calculated results due to inhomogeneous distribution of charges was also reported in Ref. 7. It is also significant that the intensity of the CP near anode is slightly larger than that of the intensity of the CP near center (Fig. 5). Since the effect of electric field is strong enough near anode in comparison with the other parts of the crystal, the randomly oriented static and dynamic PNRs may start to align in the direction of the applied field near the anode, resulting in an intense CP near the anode.

In summary, the effect of electric voltage along a [100] direction on the ferroelectric phase transition was clearly observed. The temperature-field phase diagram including CEP was clarified in the averaged field range below $3.5 \mathrm{kV} /$ $\mathrm{cm}$. The effects of trapped electrons were observed by the lower value of the CEP in comparison with the Li-doped KTN single crystal with no trapped electrons in literature. The inhomogeneous distribution of injected and/or trapped electrons in the KLTN/5/26 single crystal was discussed by the difference of the CEP between Brillouin result at a fixed small area and dielectric result averaged over all area of a sample. It can be caused by the field gradient induced by the trapped electrons, which was clarified by the observation of position dependence of the CP intensity.

This study was supported in part by JSPS KAKENHI Grant Number JP17K05030. M.M.R. is thankful to Dr. M. A. Helal for his support on experiments.

${ }^{1}$ Y. C. Chang, C. Wang, S. Yin, R. C. Hoffman, and A. G. Mott, Opt. Lett. 38, 4574 (2013).

${ }^{2}$ H. Tian, B. Yao, C. Hu, X. Meng, and Z. Zhou, Appl. Phys. Express 7, 062601 (2014). 
${ }^{3}$ A. Agranat, R. Hofmeister, and A. Yariv, Opt. Lett. 17, 713 (1992).

${ }^{4}$ K. Nakamura, J. Miyazu, Y. Sasaki, T. Imai, M. Sasaura, and K. Fujiura, J. Appl. Phys. 104, 013105 (2008).

${ }^{5}$ T. Imai, J. Miyazu, and J. Kobayashi, Opt. Express 22, 14114 (2014).

${ }^{6}$ F. S. Chen, J. E. Geusic, S. K. Kurtz, J. G. Skinner, and S. H. Wemple, J. Appl. Phys. 37, 388 (1966).

${ }^{7}$ T. Imai, S. Toyoda, J. Miyazu, J. Kobayashi, and S. Kojima, Jpn. J. Appl. Phys., Part 1 53, 09PB02 (2014).

${ }^{8}$ O. Hanske-Petitpierre, Y. Yacoby, J. Mustre de Leon, E. A. Stern, and J. J. Rehr, Phys. Rev. B 44, 6700 (1991).

${ }^{9}$ M. M. Rahaman, T. Imai, J. Kobayashi, and S. Kojima, Jpn. J. Appl. Phys., Part 1 54, 10NB01 (2015).

${ }^{10}$ J. P. Sokoloff, L. L. Chase, and L. A. Boatner, Phys. Rev. B 41, 2398 (1990).

${ }^{11}$ L. Cai, J. Toulouse, L. Harrier, R. G. Downing, and L. A. Boatner, Phys. Rev. B 91, 134106 (2015).

${ }^{12}$ R. Machado, M. Sepliarsky, and M. G. Stachiotti, Phys. Rev. B 86, 094118 (2012).
${ }^{13}$ M. M. Rahaman, T. Imai, J. Miyazu, J. Kobayashi, S. Tsukada, M. A. Helal, and S. Kojima, J. Appl. Phys. 116, 074110 (2014).

${ }^{14}$ M. M. Rahaman, T. Imai, T. Sakamoto, S. Tsukada, and S. Kojima, Ferroelectrics 487, 47 (2015).

${ }^{15}$ M. M. Rahaman, T. Imai, T. Sakamoto, S. Tsukada, and S. Kojima, Sci. Rep. 6, 23898 (2016).

${ }^{16}$ E. Dul'kin, S. Kojima, and M. Roth, Eur. Phys. Lett. 97, 57004 (2012).

${ }^{17}$ J. Toulouse, P. DiAntonio, B. E. Vugmeister, X. M. Wang, and L. A. Knauss, Phys. Rev. Lett. 68, 232 (1992).

${ }^{18}$ W. Kleemann, F. J. Schäfer, and D. Rytz, Phys. Rev. Lett. 54, 2038 (1985).

${ }^{19}$ L. A. Knauss, X. M. Wang, and J. Toulouse, Phys. Rev. B 52, 13261 (1995).

${ }^{20}$ G. Burns and F. H. Dacol, Phys. Rev. B 28, 2527 (1987).

${ }^{21}$ Z. Kutnjak, J. Petzelt, and R. Blinc, Nature 441, 956 (2006).

${ }^{22}$ M. Aftabuzzaman and S. Kojima, Jpn. J. Appl. Phys. 55, 07KB03 (2016).

${ }^{23}$ S. Kojima, Jpn. J. Appl. Phys. 49, 07HA01 (2010).

${ }^{24}$ R. Vacher and L. Boyer, Phys. Rev. B 6, 639 (1972). 